\title{
Protura from Hainan Island, China: new species, checklist and distribution
}

\author{
Yun $\mathrm{Bu}^{1,2}$, Yan Xiong ${ }^{3}$, Yun-Xia Luan ${ }^{2,4}$, Wen-Ying Yin ${ }^{2}$ \\ I Natural History Research Center, Shanghai Natural History Museum, Shanghai Science \& Technology Museum, \\ Shanghai, 200041, China 2 Institute of Plant Physiology and Ecology, Chinese Academy of Sciences, Shanghai, \\ 200032, China 3 Shanghai Information Center for Life Sciences, Shanghai Institute of Nutrition and Health, \\ Shanghai Institutes for Biological Sciences, Chinese Academy of Sciences, Shanghai 200031, China 4 Guangdong \\ Provincial Key Laboratory of Insect Developmental Biology and Applied Technology, Institute of Insect Science and \\ Technology, School of Life Sciences, South China Normal University, Guangzhou, 510631, China
}

Corresponding author: Yun-Xia Luan (yxluan@scnu.edu.cn)

Academic editor: L. Deharveng | Received 9 March 2019 | Accepted 25 August 2019 | Published 9 October 2019

http://zoobank.org/53FD6801-C4B5-4C09-B61B-47210DE937A7

Citation: Bu Y, Xiong Y, Luan Y-X, Yin W-Y (2019) Protura from Hainan Island, China: new species, checklist and distribution. ZooKeys 879: 1-21. https://doi.org/10.3897/zookeys.879.34404

\begin{abstract}
More than 1500 proturan specimens from Hainan Island are systematically studied. An annotated list of all species of Protura from Hainan Island is provided and their geographical distribution is discussed. The genus Paracondeellum is reported from Hainan Island for the first time, and Paracondeellum paradisum sp. nov. is described. The type species Paracondeellum dukouense (Tang \& Yin, 1988) is redescribed based on syntype, and the lectotype and paralectotype are designated. The characters of the genus Paracondeellum are redefined, and the two known species are compared in detail. The Protura fauna of Hainan Island is mainly composed of species from the Oriental region, with $91 \%$ of the species belonging to the families Berberentulidae and Eosentomidae.
\end{abstract}

\section{Keywords}

distribution, diversity, Paracondeellum, new species, taxonomy, type specimen

Copyright Yun Bu et al. This is an open access article distributed under the terms of the Creative Commons Attribution License (CC BY 4.0), which permits unrestricted use, distribution, and reproduction in any medium, provided the original author and source are credited. 


\section{Introduction}

Protura is a group of tiny soil-dwelling arthropods with more than 800 described species (Bu et al. 2012, 2017; Galli et al. 2018). The diagnosis, distribution, and key to 76 known genera and seven families of Protura worldwide were recently given by Galli et al. (2018). So far, there are 214 species belonging to 43 genera recorded in China (Bu et al. 2012, 2017; Qian et al. 2018).

Hainan Island is the second largest island of China and is located off the southernmost point of the mainland $\left(18^{\circ} 10^{\prime}-20^{\circ} 10^{\prime} \mathrm{N}, 108^{\circ} 37^{\prime}-111^{\circ} 03^{\prime} \mathrm{E}\right.$; Fig. 1$)$. The tropical forest landscape on Hainan Island is one of the hotspots for biodiversity in China, with a high floral diversity and over 6000 species of insects recorded (Huang 2002). In recent years, many rare insects, such as belonging to Zoraptera, have been found on Hainan Island (Yin et al. 2015).

There are several previous publications on the Protura from Hainan Island. The first study reported 14 species of Eosentomidae from Hainan (Yin 1986). Then, eight species of the genus Kenyentulus (Berberentulidae) were described (Yin 1987). Later, 24 species of Protura were recorded in Hainan Province with Fujientomon dicestum Yin, 1977 and Pseudanisentomon yongxingense Yin, 1988 included (Yin 1999, 2002). In 2004, the Protura from Jianfengling Mountain were investigated again. In 2005, Amphientulus sinensis Xiong, Xie \& Yin, 2005 was described and seven new records and three undetermined species were newly added (Xiong 2005; Xiong et al. 2005). One of these undetermined species was subsequently described as Anisentomon hainanense Xiong, $\mathrm{Bu} \&$ Yin, 2008 (Xiong et al. 2008).

In 2011 and 2017, we investigated the soil fauna of Hainan Island on several occasions and collected many proturan specimens. In the present paper, Protentomidae is recorded for the first time and one new species of genus Paracondeellum Yin, Xie \& Zhang, 1994 is identified and described. We checked the syntypes of the type species of Paracondeellum dukouense (Tang \& Yin, 1988), designated a lectotype and paralectotype, and redescribed it in detail. In addition, based on more than 1500 proturans collected in Hainan Island from 1984 to 2017, a checklist is presented and the distribution of Protura on Hainan Island is summarized.

\section{Materials and methods}

Most of specimens were collected between 1984 and 2004, and more recent specimens were collected during the expeditions in 2011 and 2017. All localities sampled so far are listed in Table 1 and shown in Figure 1. All specimens were extracted by means of the Tullgren funnels from soil and humus samples and preserved in $75 \%$ ethanol. They were mounted on slides using Hoyer's solution and dried in an oven at $50{ }^{\circ} \mathrm{C}$.

Observations were made with a phase contrast microscope (Leica DM 2500). Photos were taken by a digital camera (Leica DMC 4500). Line drawings were made using a drawing tube. All specimens are deposited in the collections of Shanghai 


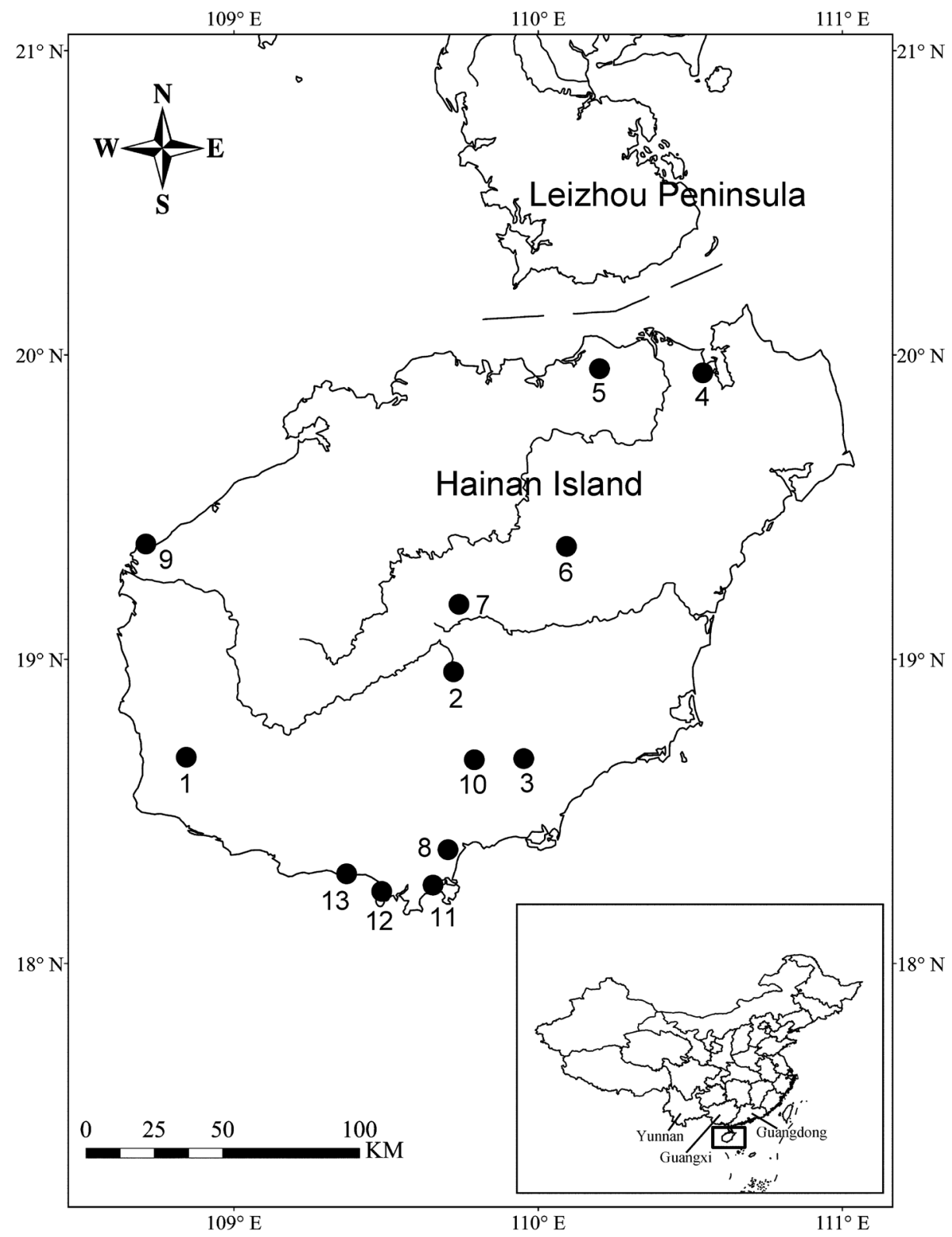

Figure I. The sampling localities in Hainan Island. Numbers 1-13 indicate the localities listed in Tables 1, 5.

Natural History Museum (SNHM) and Shanghai Entomological Museum (SEM), Shanghai, China.

Abbreviations used in the text follow the paper by $\mathrm{Bu}$ and Yin (2007). Head setae and pores are named according to Rusek et al. (2012) and Shrubovych (2014). The arrangement of the taxa follows the system proposed by Yin (1999). 
Table I. The sampling localities of Protura in Hainan Island.

\begin{tabular}{llccc}
\hline Number & Locality & Coordinates & Altitude (m) & Sampling years \\
\hline $\mathbf{1}$ & Ledong County, Jianfengling National Natural Reserve & $18^{\circ} 23^{\prime}-18^{\circ} 52^{\prime} \mathrm{N}$, & $120-330$ & $1984,1993,2003,2004$ \\
& & $108^{\circ} 44^{\prime}-109^{\circ} 02^{\prime} \mathrm{E}$ & & \\
$\mathbf{2}$ & Wuzhishan City, Wuzhishan National Natural Reserve & $18^{\circ} 49^{\prime}-18^{\circ} 59^{\prime} \mathrm{N}$, & $800-1200$ & $1984,1985,2004,2011$ \\
& & $109^{\circ} 32^{\prime}-109^{\circ} 43^{\prime} \mathrm{E}$ & & \\
$\mathbf{3}$ & Wuzhishan City, Diaoluoshan National Natural Reserve & $18^{\circ} 43^{\prime}-18^{\circ} 58^{\prime} \mathrm{N}$, & $500-1000$ & 1985,2004 \\
& & $109^{\circ} 43^{\prime}-110^{\circ} 03^{\prime} \mathrm{E}$ & & 2004 \\
$\mathbf{4}$ & Haikou City, Dongzhaigang National Natural Reserve & $19^{\circ} 51^{\prime}-20^{\circ} 01^{\prime} \mathrm{N}$, & 20 & 2003 \\
$\mathbf{5}$ & Haikou City, Crater National Geological Park & $110^{\circ} 32^{\prime}-110^{\circ} 37^{\prime} \mathrm{E}$ & & 2003 \\
$\mathbf{6}$ & Tunchang County, Meiling Mountain & $19^{\circ} 55^{\prime} \mathrm{N}, 110^{\circ} 12^{\prime} \mathrm{E}$ & 223 & 2003 \\
$\mathbf{7}$ & Tunchang County, Limu Mountain & $19^{\circ} 22^{\prime} \mathrm{N}, 110^{\circ} 04^{\prime} \mathrm{E}$ & $150-230$ & 2003 \\
$\mathbf{8}$ & Baoting County, Ganshenling Provincial Natural Reserve & $19^{\circ} 17^{\prime} \mathrm{N}, 109^{\circ} 77^{\prime} \mathrm{N}, 109^{\circ} 66^{\prime} \mathrm{E}$ & $600-1000$ & 500 \\
$\mathbf{9}$ & Changjiang County, Qizi bay & $19^{\circ} 21^{\prime} \mathrm{N}, 108^{\circ} 40^{\prime} \mathrm{E}$ & 15 & 2011 \\
$\mathbf{1 0}$ & Baoting, Qixianling National Forest Park & $18^{\circ} 42^{\prime} \mathrm{N}, 109^{\circ} 40^{\prime} \mathrm{E}$ & 150 & 2017 \\
$\mathbf{1 1}$ & Sanya City, Yalong Bay Tropical Paradise Forest Park & $18^{\circ} 15^{\prime} \mathrm{N}, 109^{\circ} 38^{\prime} \mathrm{E}$ & 200 & 2017 \\
$\mathbf{1 2}$ & Sanya City, Luhuitou Park & $18^{\circ} 13^{\prime} \mathrm{N}, 109^{\circ} 29^{\prime} \mathrm{E}$ & 80 & 2017 \\
$\mathbf{1 3}$ & Sanya City, Sanya bay & $18^{\circ} 17^{\prime} \mathrm{N}, 109^{\circ} 22^{\prime} \mathrm{E}$ & 5 & 2017 \\
\hline
\end{tabular}

\section{Results}

\section{Taxonomy}

\section{Family Protentomidae Ewing, 1936}

\section{Genus Paracondeellum Yin, Xie \& Zhang, 1994}

Diagnosis. Habitus short and robust. Pseudoculi circular without lever. Calyx of maxillary glands globular and smooth. Foretarsal sensilla of the exterior side reduced; interior sensilla $b$ ' absent. Abdominal appendages I-II two-segmented each with four setae, III uni-segmented with two setae. Tergites II-VII without or with few anterior setae. Sternites II-III each with three posterior setae. Sternites IV-VII each with nine posterior setae; sternite VIII with four setae in one row. Female squama genitalis short, with pointed acrostyli (Yin 1999; Galli et al. 2018).

Distribution. South China (Sichuan, Yunnan, Hainan).

Remarks. Paracondeellum Yin, Xie \& Zhang, 1994 was originally separated from the genus Condeellum Tuxen, 1963. They have similar shapes of pseudocellus and maxillary gland, and the presence of setae $P c$ on sternites IV-V, but they can be easily separated by the chaetotaxy of tergite I (seta P5 absent in Paracondeellum but present in Condeellum) and sternite VIII (four setae in Paracondeellum vs six setae in Condeellum). In addition, Paracondeellum can be distinguished from the genus Neocondeellum Tuxen \& Yin, 1982 by the shape of pseudocellus (posterior lever absent in Paracondeellum but present in Neocondeellum) and the chaetotaxy of sternites IV-V (setae Pc present in Paracondeellum but absent in Neocondeellum). 


\section{Paracondeellum paradisum Bu \& Yin, sp. nov.}

http://zoobank.org/A723F8F3-18BF-420F-885E-29EA34F782D7

Figures 2-4; Tables 2, 4

Diagnosis. Paracondeellum paradisum sp. nov. is characterized by two pairs of $A$-setae on tergite I, one pair of $A$-setae, and eight pairs of $P$-setae on tergites II-VI, absence of $A$-setae and $P 2 a$ seta on tergite VII, tergites IX and X with 12 and 10 setae, respectively, absence of seta $d 4$ on dorsal side of head, and female squama genitalis short, with conical acrostylus.

Material examined. Holotype, female (slide no. HN-SY-P2017016) (SNHM), China, Hainan, Sanya City, Yalong Bay Tropical Paradise Forest Park, soil of the tropical rain forest, $200 \mathrm{~m}$ elev., $18.25^{\circ} \mathrm{N}, 109.63^{\circ} \mathrm{E}, 22-\mathrm{III}-2017$, Y. Bu collector. Paratypes, 1 female (slide no. HN-SY-P2017071) (SNHM), same data as holotype.

Description. Holotype: body length $570 \mu \mathrm{m}$, yellow-brown, foretarsus darker (Fig. 4A).

Head. Elliptic, length $80 \mu \mathrm{m}$, width $50 \mu \mathrm{m}$ (Fig. 2A). Head setae short, rostrum slightly protruded. Setae $d \sigma$ and $s d \sigma$ present, $d 4$ and $s d 4$ absent, $d \sigma$ and $d 7$ length $\sigma \mu \mathrm{m}$ and $7 \mu \mathrm{m}$ respectively. Pores $c p$ and $f p$ present. Pseudoculus oval, without lever, length $8 \mu \mathrm{m}$, width $6.5 \mu \mathrm{m}$. $\mathrm{PR}=10$ (Fig. $2 \mathrm{~B}$ ). Canal of maxillary gland short, with globular calyx and short sausage-like posterior dilation. $\mathrm{CF}=10$ (Figs 2C, 4B). Labial palpus well developed, with four setae and apical tuft, without basal sensillum (Fig. 2D). Maxillary palpus with two subequal seta-like sensilla (Fig. 2E).

Foretarsus. Length $31 \mu \mathrm{m}$, claw length $9 \mu \mathrm{m}, \mathrm{TR}=3.4$; empodium length $2 \mu \mathrm{m}$, $\mathrm{EU}=0.22$. Dorsal sensilla $t-1$ and $t-2$ slender and long, $\mathrm{BS}=0.63 ; t-3$ short and spatulate, not reaching base of claw (Fig. 2G). Exterior side with only sensilla $a, b$ and $f$ present; $a$ spatulate, $b$ and $f$ short (Fig. 2 F). Interior sensilla a' and $c$ 'short sward-like, $b^{\prime}$ absent. Relative length of sensilla: $t-2>t-1>c^{\prime}>t 3>a>a^{\prime}>(b=f)$ (Fig. 2F, G). Length of middle tarsus $15 \mu \mathrm{m}$; claw length $10 \mu \mathrm{m}$. Length of hind tarsus $17 \mu \mathrm{m}$; claw length $12 \mu \mathrm{m}$.

Thorax. Thoracic chaetotaxy given in Table 2. Setae 1 and 2 on pronotum subequal in length, $6 \mu \mathrm{m}$ and $7 \mu \mathrm{m}$ respectively (Fig. $2 \mathrm{H}$ ); mesonotum with seven pairs of posterior setae, $P 5 a$ minute; metanotum with six pairs of posterior setae, $P 5 a$ absent; setae $P 1, P 1 a, P 2$ on mesonotum $6 \mu \mathrm{m}, 1 \mu \mathrm{m}, 7 \mu \mathrm{m}$, respectively; P1 1 on meso- and metanotum short, pin-shaped (Fig. 2H). Prosternum without seta $A 2$. All setae on thoracic sternites of normal shape. Pores on thorax not observed.

Abdomen. Abdominal chaetotaxy given in Table 2. Tergite I with two pairs of anterior setae $(A 1, A 5)$ and six pairs of posterior setae, $A 5$ short, sensillum-shaped (Fig. 2I). Tergites II-VI with one pair of anterior $(A I)$ and eight pairs of posterior setae, $P 2 a$ present and $P 3 a$ absent (Figs 2J, 3A, 4E, F). Tergite VII without anterior setae and with eight posterior setae, $P 2 a$ absent and P3a present (Figs 3B, C, 4E, F). Accessory setae Pla on tergites I-V short pin-shaped $(4 \mu \mathrm{m})$, on tergites VI-VII normal $(5 \mu \mathrm{m})$. Accessory setae P2a and P4a always pin-shaped, $2 \mu \mathrm{m}$ in length. P3a on tergite VII 


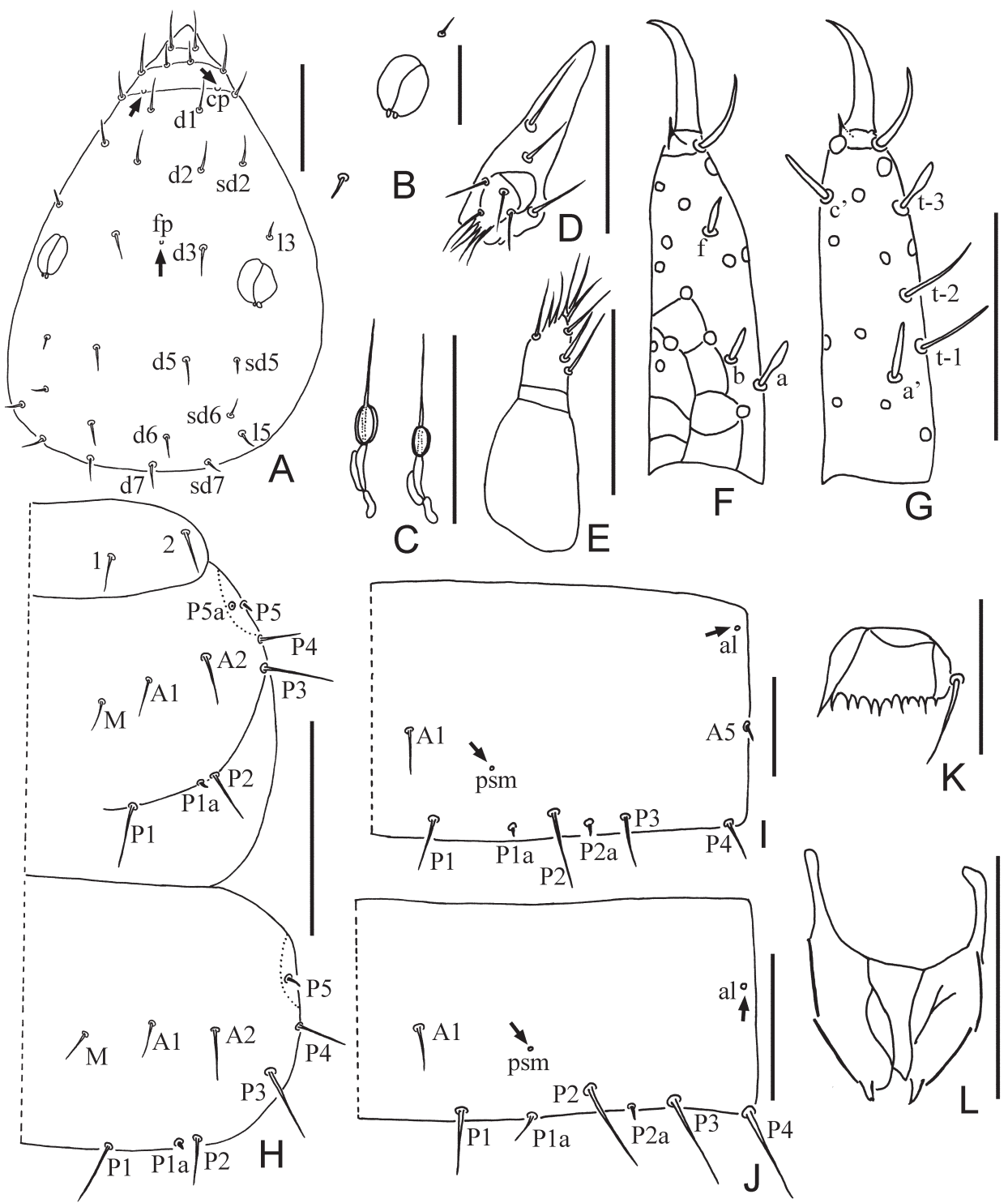

Figure 2. Paracondeellum paradisum sp. nov., holotype $\mathbf{A}$ head, dorsal view ( $c p=$ clypeal pore, $f p=$ frontal pore) $\mathbf{B}$ pseudoculus $\mathbf{C}$ canal of maxillary gland $\mathbf{D}$ labial palpus $\mathbf{E}$ maxillary palpus $\mathbf{F}$ foretarsus, exterior view $\mathbf{G}$ foretarsus, interior view $\mathbf{H}$ dorsal thorax, right side $\mathbf{I}$ tergite I, right side ( $a l=$ anterolateral pore, $p s m=$ posterosubmedial pore) $\mathbf{J}$ tergite $\mathrm{VI}$, right side $\mathbf{K} \operatorname{comb} \mathbf{L}$ female quama genitalis. Arrows indicate pores. Scale bars: $10 \mu \mathrm{m}(\mathbf{B}, \mathbf{K}) ; 20 \mu \mathrm{m}(\mathbf{A}, \mathbf{C}-\mathbf{J}, \mathbf{L})$.

of normal shape and $5 \mu \mathrm{m}$ long (Fig. 4E, F). Tergite VIII with two pairs of anterior setae (A1, A3) (Fig. 4C). Posterior central seta Pc present on sternites IV-VII, sensillum shaped, 4-5 $\mu \mathrm{m}$ long (Figs 3A, C, 4F). P1a on sternites IV-VI short, pin-shaped (Fig. 3A), on sternite VII setiform (Fig. 3C). 


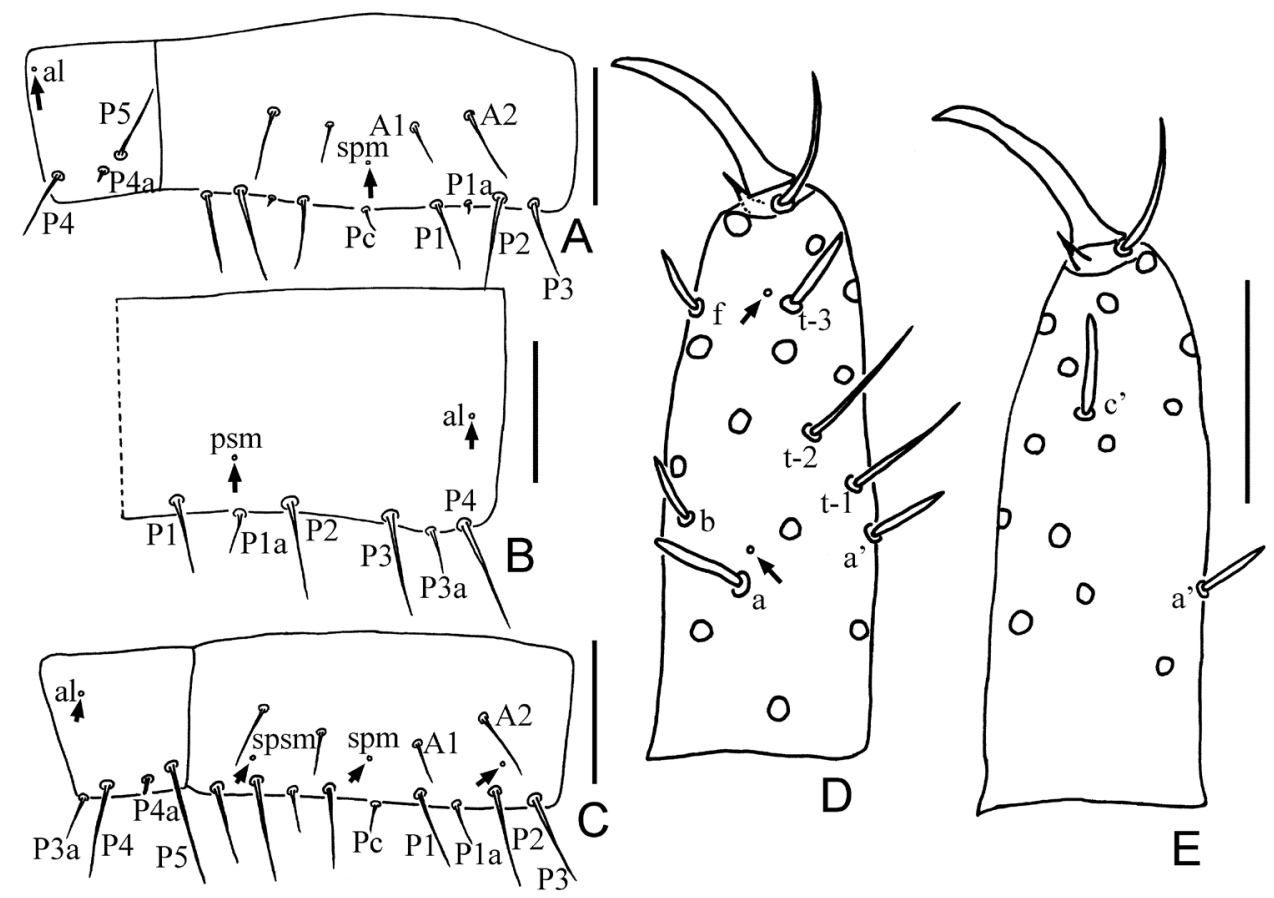

Figure 3. A-C Paracondeellum paradisum sp. nov., holotype $\mathbf{A}$ sternite VI (spm = sternal posteromedial pore) B tergite VII, right side $\mathbf{C}$ sternite VII (spsm = sternal posterosubmedial pore). D-E Paracondeellum dukouense (Tang \& Yin, 1988) holotype $\mathbf{D}$ foretarsus, exterior view E foretarsus, interior view. Arrows indicate pores. Scale bars: $20 \mu \mathrm{m}$.

Tergites I-VII with pores psm and al (Fig. 2I, J), VIII with pores psm only, IX-XI without pores, XII single median pore. Sternites I-VI each with single posteromedial pore spm (Figs 3A, 4F), VII with three posteromedial pores (Figs 3C, 4F), VIII with single posteromedial pore (Fig. 4D), IX-XI without pores, XII with one pair of anterolateral sal pores.

Abdominal appendages typical of the genus. Subapical setae and apical setae on appendage III $11 \mu \mathrm{m}$ and $5 \mu \mathrm{m}$ long respectively. Striate band on abdominal segment VIII reduced to a single serrate line (Fig. 4D). Comb on abdomen VIII rectangular, with 10 teeth, $10 \mu \mathrm{m}$ wide (Fig. $2 \mathrm{~K}$ ). Female squama genitalis short, with conical acrostylus (Fig. 2L).

Etymology. Latin "paradisum", after "Paradise Forest Park" where type specimens were collected.

Distribution. China (Hainan)

Remarks. The genus Paracondeellum Yin, Xie \& Zhang, 1994 is endemic to China and was previously known by a single species, $P$. dukouense, from Sichuan and Yunnan provinces. Paracondeellum paradisum sp. nov. differs from $P$. dukouense in the shape of foretarsal sensilla, pseudoculus, and female squama genitalis, and in the body chaetotaxy. A comparison of the morphology of these two species is given in Table 4. 
Table 2. Adult chaetotaxy of Paracondeellum paradisum sp. nov.

\begin{tabular}{|c|c|c|c|c|c|}
\hline \multirow{2}{*}{\multicolumn{2}{|c|}{ Segment }} & \multicolumn{2}{|c|}{ Dorsal } & \multicolumn{2}{|c|}{ Ventral } \\
\hline & & Formula & Setae & Formula & Setae \\
\hline \multirow{6}{*}{ Th. } & \multirow{2}{*}{ I } & \multirow{2}{*}{4} & \multirow{2}{*}{1,2} & \multirow{2}{*}{$(2+2) / 6$} & $A 1, M$ \\
\hline & & & & & $P 1,2,3$ \\
\hline & \multirow{2}{*}{ II } & \multirow{2}{*}{$6 / 14$} & $A 2,4, M$ & \multirow{2}{*}{$(4+2) / 4$} & $A 1,2, M$ \\
\hline & & & $P 1,1 a, 2,3,4,5,5 a$ & & $P 1,2$ \\
\hline & \multirow{2}{*}{ III } & \multirow{2}{*}{$6 / 12$} & $A 2,4, M$ & \multirow{2}{*}{$(6+2) / 4$} & $A 1,2,3, M$ \\
\hline & & & $P 1,1 a, 2,3,4,5$ & & $P 1,2$ \\
\hline \multirow{14}{*}{ Abd. } & \multirow{2}{*}{ I } & \multirow{2}{*}{$4 / 12$} & $A 1,5$ & \multirow{2}{*}{$4 / 2$} & $A 1,2$ \\
\hline & & & $P 1,1 a, 2,2 a, 3,4$ & & P1 \\
\hline & \multirow{2}{*}{ II-III } & \multirow{2}{*}{$2 / 16$} & $A 1$ & \multirow{2}{*}{$4 / 3$} & $A 1,2$ \\
\hline & & & $P 1,1 a, 2,2 a, 3,4,4 a, 5$ & & $P c, 2$ \\
\hline & \multirow{2}{*}{ IV-VI } & \multirow{2}{*}{$2 / 16$} & $A 1$ & \multirow{2}{*}{$4 / 9$} & $A 1,2$ \\
\hline & & & $P 1,1 a, 2,2 a, 3,4,4 a, 5$ & & $P c, 1,1 a, 2,3$ \\
\hline & \multirow{2}{*}{ VII } & \multirow{2}{*}{$0 / 16$} & & \multirow{2}{*}{$4 / 9$} & $A 1,2$ \\
\hline & & & $P 1,1 a, 2,3,3 a, 4,4 a, 5$ & & $P c, 1,1 a, 2,3$ \\
\hline & \multirow{2}{*}{ VIII } & \multirow{2}{*}{$4 / 14$} & $A 1,3$ & & \\
\hline & & & $P 1,1 a, 2,2 a, 3,3 a, 4$ & 4 & 1,2 \\
\hline & IX & 12 & $1,1 a, 2,2 a, 3,4$ & 4 & 1,2 \\
\hline & $\mathrm{X}$ & 10 & $1,2,2 a, 3,4$ & 4 & 1,2 \\
\hline & $\mathrm{XI}$ & 6 & & 6 & $1,2,3$ \\
\hline & XII & 9 & & 6 & \\
\hline
\end{tabular}

\section{Paracondeellum dukouense (Tang \& Yin, 1988)}

Figures 3, 5; Tables 3, 4

Diagnosis. Paracondeellum dukouense (Tang \& Yin, 1988) is characterized by the one pair of $A$-setae on tergite I, absence of $A$-setae and Pla seta on tergites II-VI, absence of $A$-setae and nine pairs of $P$-setae (P2a present) on tergite VII, tergites IX and X with 14 and 12 setae respectively, absence of seta $d 4$ on head, and female squama genitalis with pointed acrostylus.

Material examined. Lectotype, female (slide no. 1), paralectotype, female (slide no. 2) (SEM), China, Sichuan, Dukou City (currently, Panzhihua City), Jinjiang County, soil under grass, 1155 m elev., 26.55N, 101.85E, 26-IX-1985, B.W. Tang and G.T. Jin collectors. We designated as the lectotype the female on slide no. 1 and the other female on slide no. 2 as the paralectotype.

Redescription. Body length of holotype $880 \mu \mathrm{m}$ and paratype $720 \mu \mathrm{m}$; yellowbrown, with foretarsus darker (Fig. 5A).

Head. Elliptic, length 93-100 $\mu \mathrm{m}$, width $70 \mu \mathrm{m}$. Dorsal setae longer than subdorsal and lateral ones, rostrum slightly protruded (Fig. 5C). Setae $d \sigma$ and $s d 6$ present, $s d \sigma$ sensillum-shaped; $d 4$ and $s d 4$ absent; $d 6$ and $d 711 \mu \mathrm{m}$ and $6 \mu \mathrm{m}$ long, respectively (Fig. 5C). Pores $c p$ and $f p$ present. Pseudoculus round, without lever, length $13 \mu \mathrm{m}$, width $11 \mu \mathrm{m}$. PR = 7.2-7.7 (Fig. 5C). Canal of maxillary gland short, with globular calyx and sausage-like posterior dilation. $\mathrm{CF}=13.3-14.3$ (Fig. 5B). Labial palpus well developed, with four setae and apical tuft, without basal sensillum. Maxillary palpus with two subequal sensilla.

Foretarsus. Length 46-50 $\mu \mathrm{m}$, claw length $15-17 \mu \mathrm{m}, \mathrm{TR}=2.9-3.1$; empodium length $4-5 \mu \mathrm{m}, \mathrm{EU}=0.24-0.33$. Dorsal sensilla $t-1$ and $t-2$ slender and long, $\mathrm{BS}=0.66$; 


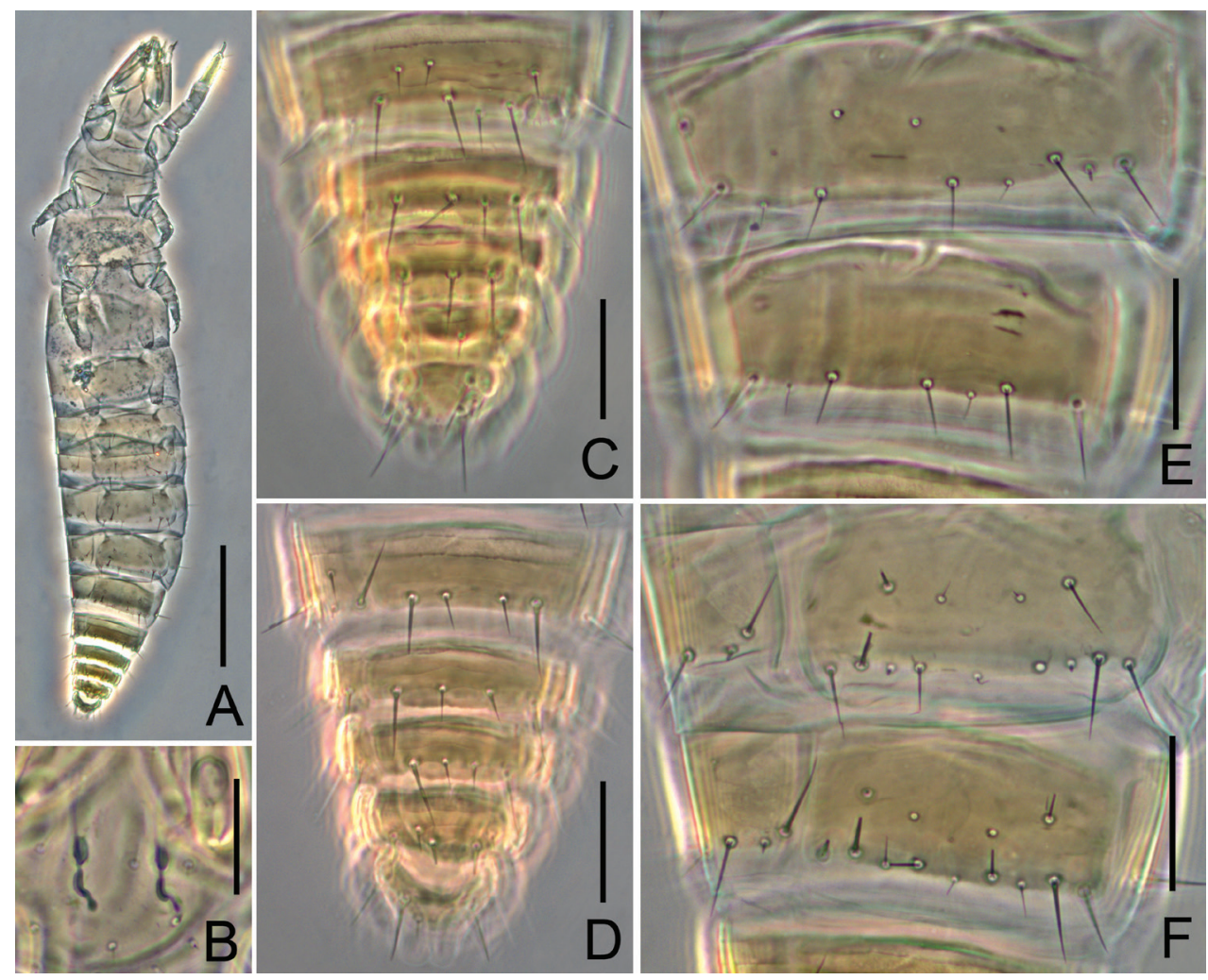

Figure 4. Paracondeellum paradisum sp. nov., holotype $\mathbf{A}$ habitus $\mathbf{B}$ canal of maxillary gland $\mathbf{C}$ tergites VIII-XII D sternites VIII-XII E tergites V-VII F sternites VI-VII. Scale bars: $100 \mu \mathrm{m}(\mathbf{A}) ; 20 \mu \mathrm{m}(\mathbf{B}-\mathbf{F})$.

$t-3$ short sward-like, nearly reaching base of claw (Fig. 3D). Exterior slide with only sensilla $a, b$ and $f$ present; $a$ spatulate, $b$ and $f$ short sward-like (Fig. 3D). Interior sensilla $a$ ' and $c$ 'short sward-like, $b$ 'absent (Fig. 3E). Relative length of sensilla: $t-2>t-1>$ $c^{\prime}>a>t 3>a^{\prime}>(b=f)$ (Fig. 3D, E). Length of middle tarsus $20 \mu \mathrm{m}$; claw length 12 $\mu \mathrm{m}$. Length of hind tarsus $23 \mu \mathrm{m}$; claw length $15 \mu \mathrm{m}$.

Thorax. Thoracic chaetotaxy given in Table 3. Setae 1 and 2 on pronotum subequal in length, $10 \mu \mathrm{m}$ long; mesonotum with seven pairs of posterior setae, $P 5 \mathrm{a}$ minute; metanotum with six pairs of posterior setae, P5a absent; setae P1, P1a, P2 on mesonotum $10 \mu \mathrm{m}, 1.5 \mu \mathrm{m}, 14 \mu \mathrm{m}$ respectively; P1a on meso- and metanotum short, pin-shaped. Prosternum with anterior seta $A 2$ (Fig. 5D), meso- and metasternum each with four posterior setae (Fig. 5E), metasternum with six anterior setae. All setae on sterna normal. Pores on thorax not detectable.

Abdomen. Abdominal chaetotaxy given in Table 3. Tergite I with one pair of anterior setae $(A 5)$ and six pairs of posterior setae, $A 5$ short, sensillum-shaped. Tergites II-VI without anterior setae and seven pairs of posterior setae, $P 2 a$ present, $P 1 a$ and $P 3 a$ absent (Fig. 5F). Tergite VII without anterior setae and with nine pairs of posterior setae, both $P 2 a$ and P3a present (Fig. 5G). Accessory setae P2a and P4a on tergites II-VII short, sensillum-shaped, $4 \mu \mathrm{m}$ in length, P1a and P3a on tergites VII normal, 


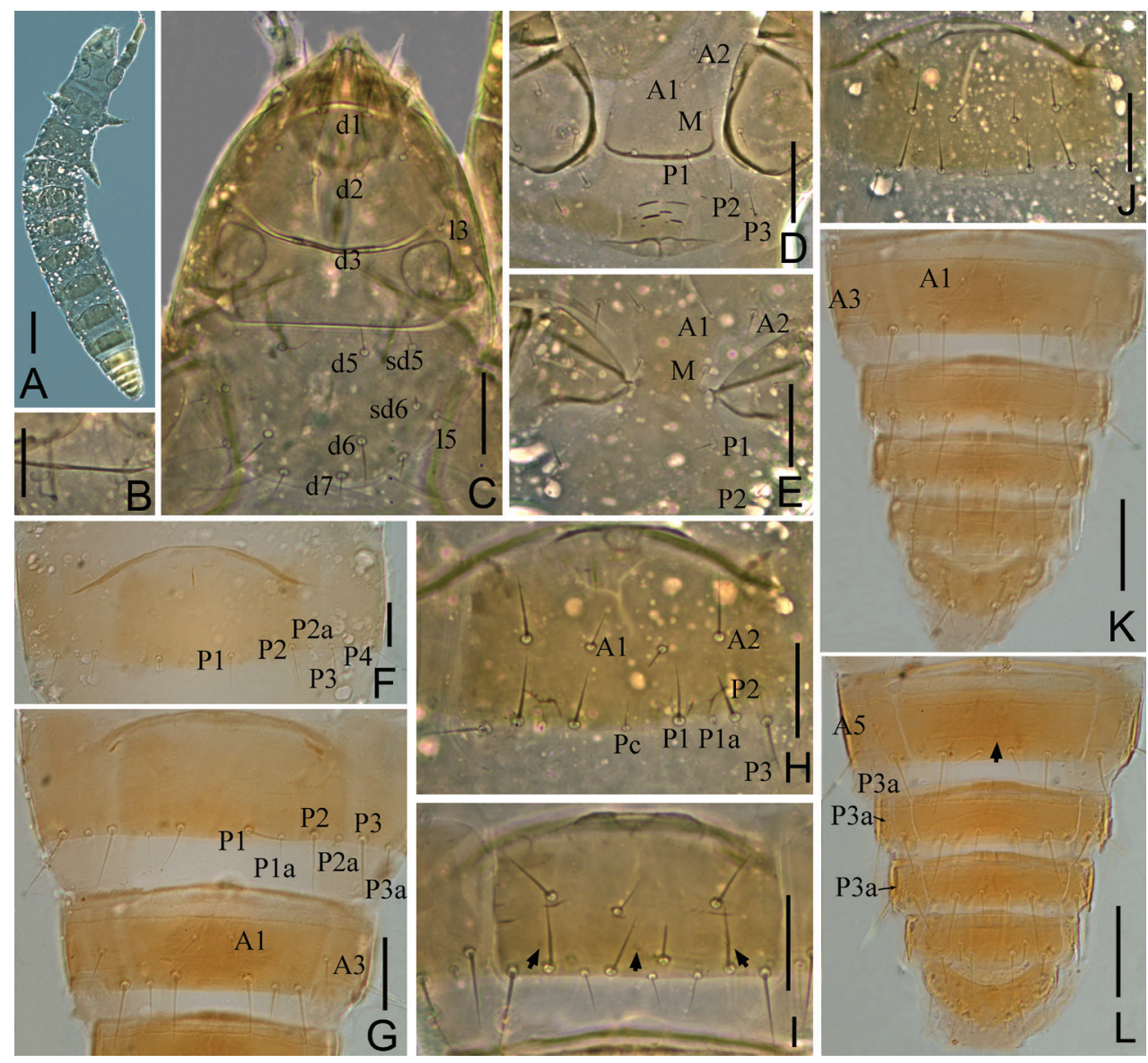

Figure 5. Paracondeellum dukouense (Tang \& Yin, 1988), holotype A habitus B canal of maxillary gland $\mathbf{C}$ head, dorsal view $\mathbf{D}$ prosternum $\mathbf{E}$ mesosternum $\mathbf{F}$ tergite VI $\mathbf{G}$ tergites VII-VIII $\mathbf{H}$ sternite VI I sternite VII J sternite V K tergites VIII-XII L sternites VIII-XII. Arrows indicate pores. Scale bars: $20 \mu \mathrm{m}$.

9-10 $\mu \mathrm{m}$ in length (Fig. 5F, G). Tergite VIII with three pairs of anterior setae ( $A 1$, $A 3, A 5)$ and seven pairs of posterior setae, P3a short $(5 \mu \mathrm{m})$ (Fig. $5 \mathrm{G}, \mathrm{K}, \mathrm{L})$. Posterior central seta $P c$ present on sternites IV-VII slender, $8-9 \mu \mathrm{m}$ long (Fig. 5H-J). P1a on sternites IV-VI short pin-shaped, $2 \mu \mathrm{m}$ long (Fig. $5 \mathrm{H}$, J), on sternite VII as normal seta, $9 \mu \mathrm{m}$ long (Fig. 5I). Sternites IX and X with short P3a seta (Fig. 5L), which had been omitted in original description.

Tergites I-VII with pores $p s m$ and $a l$, VIII with pores psm only, IX-XI without pores, XII with single median pore. Pores on sternites I-VI not observed due to the opacity of the old specimens (Fig. $5 \mathrm{H}, \mathrm{J}$ ); three posteromedial pores observed on sternite VII (Fig. 5I), VIII with posteromedial pore (Fig. 5L), IX-XI without pores, XII with one pair of sal pore.

Abdominal appendages typical of the genus. Subapical setae and apical setae on appendage III $12-13 \mu \mathrm{m}$ and $6-8 \mu \mathrm{m}$ long, respectively. Striate band on abdominal segment VIII reduced to a single serrate line (Fig. 5G, K, L). Comb on abdomen VIII 
Table 3. Adult chaetotaxy of Paracondeellum dukouense (Tang \& Yin, 1988).

\begin{tabular}{|c|c|c|c|c|c|}
\hline \multirow{2}{*}{\multicolumn{2}{|c|}{ Segment }} & \multicolumn{2}{|r|}{ Dorsal } & \multicolumn{2}{|c|}{ Ventral } \\
\hline & & Formula & Setae & Formula & Setae \\
\hline \multirow[t]{6}{*}{ Th. } & \multirow[t]{2}{*}{ I } & \multirow[t]{2}{*}{4} & 1,2 & \multirow[t]{2}{*}{$(4+2) / 6$} & $A 1,2, M$ \\
\hline & & & & & $P 1,2,3$ \\
\hline & \multirow[t]{2}{*}{ II } & \multirow[t]{2}{*}{$6 / 14$} & $A 2,4, M$ & \multirow[t]{2}{*}{$(4+2) / 4$} & $A 1,2, M$ \\
\hline & & & $P 1,1 a, 2,3,4,5,5 a$ & & $P 1,2$ \\
\hline & \multirow[t]{2}{*}{ III } & \multirow[t]{2}{*}{$6 / 12$} & $A 2,4, M$ & \multirow[t]{2}{*}{$(6+2) / 4$} & $A 1,2,3, M$ \\
\hline & & & $P 1,1 a, 2,3,4,5$ & & $P 1,2$ \\
\hline \multirow[t]{14}{*}{ Abd. } & \multirow[t]{2}{*}{ I } & \multirow[t]{2}{*}{$2 / 12$} & $A 5$ & \multirow[t]{2}{*}{$4 / 2$} & $A 1,2$ \\
\hline & & & $P 1,1 a, 2,2 a, 3,4$ & & P1 \\
\hline & \multirow[t]{2}{*}{ II-III } & \multirow[t]{2}{*}{$0 / 14$} & & \multirow[t]{2}{*}{$4 / 3$} & $A 1,2$ \\
\hline & & & $P 1,2,2 a, 3,4,4 a, 5$ & & Pc, 2 \\
\hline & \multirow[t]{2}{*}{ IV-VI } & \multirow[t]{2}{*}{$0 / 14$} & & \multirow[t]{2}{*}{$4 / 9$} & $A 1,2$ \\
\hline & & & $P 1,2,2 a, 3,4,4 a, 5$ & & $P c, 1,1 a, 2,3$ \\
\hline & \multirow[t]{2}{*}{ VII } & \multirow[t]{2}{*}{$0 / 18$} & & \multirow[t]{2}{*}{$4 / 9$} & $A 1,2$ \\
\hline & & & $P 1,1 a, 2,2 a, 3,3 a, 4,4 a, 5$ & & $P c, 1,1 a, 2,3$ \\
\hline & \multirow[t]{2}{*}{ VIII } & \multirow[t]{2}{*}{$6 / 14$} & $A 1,3,5$ & & \\
\hline & & & $P 1,1 a, 2,2 a, 3,3 a, 4$ & 4 & 1,2 \\
\hline & IX & 14 & $1,1 a, 2,2 a, 3,3 a, 4$ & 4 & 1,2 \\
\hline & $\mathrm{X}$ & 12 & $1,2,2 a, 3,3 a, 4$ & 4 & 1,2 \\
\hline & $\mathrm{XI}$ & 6 & & 6 & $1,2,3$ \\
\hline & XII & 9 & & 6 & \\
\hline
\end{tabular}

Table 4. Comparison between Paracondeellum paradisum sp. nov. and $P$. dukouense (Tang $\&$ Yin, 1988).

\begin{tabular}{lcc}
\hline & Paracondeellum paradisum sp. nov. & P. dukouense \\
\hline body length $(\mu \mathrm{m})$ & 570 & $720-880$ \\
pseudoculus $(\mu \mathrm{m})$ & 8 & 13 \\
foretarsus $(\mu \mathrm{m})$ & 31 & $46-50$ \\
sensilla $b$ and $f$ & short, rod-like & longer, sward-like \\
sensillum $t-3$ & short and spatulate & longer, sward-like \\
$A$-setae on tergite I & $4(A 1, A 5)$ & $2(A 5)$ \\
$A$-setae on tergites II-VI & $2(A 1)$ & 0 \\
$P$-setae on tergites II-VI & $16(P 1 a$ present $)$ & $14(P 1 a$ absent $)$ \\
$P$-setae on tergite VII & $16(P 2 a$ absent $)$ & $18(P 2 a$ present $)$ \\
$A$-setae on tergite VIII & $4(A 1, A 3)$ & $6(A 1, A 3, A 5)$ \\
setae on tergite IX & $12(P 3 a$ absent $)$ & $14(P 3 a$ present $)$ \\
setae on tergite X & $10(P 3 a$ absent $)$ & $12(P 3 a$ present $)$ \\
$A$-setae on prosternum & $2(A 2$ absent $)$ & $4(A 2$ present $)$ \\
\hline
\end{tabular}

rectangular, with 10 teeth, $12-13 \mu \mathrm{m}$ wide (Fig. 5K). Female squama genitalis short, with pointed acrostylus.

Etymology. Named for Dukou City (now Panzhihua City, Sichuan Province) where type specimens were collected.

Distribution. China (Sichuan, Yunnan).

Remarks. Paracondeellum dukouense was originally described based on two syntypes (Tang and Yin 1988). In the original description (Tang and Yin 1988) and in the monograph of Yin (1999), most important characters such as foretarsal sensilla, pseudoculus, maxillary gland, as well as body chaetotaxy were briefly described and illustrated. After careful study of type specimens under a modern phase contrast microscope with higher resolution, we find that sensillum $c$ ' is present on the foretarsus and 
that some of the setae on the body were previously ignored due to the lower resolution of the microscope used. We correct here these mistakes in the original description and supplement the description of head chaetotaxy, the porotaxy, and the shapes of setae on the body. Table 4 compares $P$. dukouense with the new species.

\section{List of species from Hainan Island \\ Family Protentomidae Ewing, 1936}

Paracondeellum paradisum sp. nov.

Description. The description is given above.

\section{Family Berberentulidae Yin, 1983}

\section{Baculentulus tienmushanensis (Yin, 1963)}

Material examined. 1 male, $1 \mathrm{mj}$, locality 3, 19-I-1985, coll. G. T. Jin \& Z. Y. Liu. 4 females, $1 \mathrm{mj}$, locality 5, 26-II-2003; 1 female, locality 1, 14-I-2004; 10 females, $6 \mathrm{mj}$, locality 2, 27-I-2004, coll. Y. Xiong. $1 \mathrm{mj}$, locality 11, 22-III-2017, coll. Y. Bu.

Distribution. Widely distributed in China (Hainan, Zhejiang, Shanghai, Jiangxi, Anhui, Hubei, Sichuan, Chongqing, Guizhou, Yunnan, Ningxia, Gansu, Shaanxi, Henan, Hebei, Liaoning, Neimenggu).

\section{Kenyentulus ciliciocalyci Yin, 1987}

Material examined. 5 females, locality 1, 27-XI-1984, coll. G. T. Jin \& Z. Y. Liu. 1 female, VI-1993; 1 female, IV-1994, locality 1, coll. C. H. Liao. 9 females, 2 males, locality 5, 26-II-2003; 1 female, locality 6, 2-III-2003; 1 female, locality 5, 15-VI2003; 6 females, 1 male, locality 1, 6-X-2003; 9 females, 2 males, $13 \mathrm{mj}$, locality 1 , 14-I-2004; 7 females, 3 males, locality 1, 15-I-2004; 1 female, locality 1, 14-IV-2004; 2 females, 5 males, $1 \mathrm{mj}$, locality 1, 15-VII-2004, coll. Y. Xiong. 2 females, 1 male, 2 mj, locality 9, 20-III-2011, coll. Y. Bu \& C. W. Huang. 14 females, 10 male, $1 \mathrm{mj}$, locality 11, 22-III-2017; 3 males, locality 12, 17-X-2017, coll. Y. Bu.

Distribution. Widely distributed in China (Hainan, Zhejiang, Hunan, Sichuan, Chongqing, Guizhou, Yunnan, Shaanxi).

Kenyentulus dolichadeni Yin, 1987

Material examined. 3 females, locality 2, 14-XI-1984, coll. G. T. Jin \& Z. Y. Liu. 
Distribution. China (Hainan, Zhejiang, Guangxi, Guizhou, Hubei, Sichuan, Jiangxi).

\section{Kenyentulus hainanensis Yin, 1987}

Material examined. 4 females, 1 male, locality 1, 30-XI-1984; 2 females, 2 males, locality 2, 14-XI-1984; 1 male, 2 mj, locality 3, 19-I-1985, coll. G. T. Jin \& Z. Y. Liu. Distribution. China (Hainan, Guangdong).

Kenyentulus henanensis Yin, 1983

Material examined. 2 female, 1 male, locality 2, 19-XI-1984, coll. G. T. Jin \& Z. Y. Liu.

Distribution. Widely distributed in China (Hainan, Zhejiang, Jiangxi, Henan, Hubei, Guizhou, Yunnan, Ningxia).

\section{Kenyentulus japonicus (Imadate, 1961)}

Material examined. 2 females, locality 2, 14-XI-1984; 5 females, 5 males, locality 1 , 25-XI-1984, coll. G. T. Jin \& Z. Y. Liu.

Distribution. Widely distributed in China (Hainan, Zhejiang, Jiangsu, Shanghai, Jiangxi, Anhui, Hunan, Sichuan, Guizhou, Yunnan, Shaanxi); Japan.

\section{Kenyentulus jianfengensis Yin, 1987}

Material examined. 4 females, locality 1, 1-XII-1984, coll. G. T. Jin \& Z. Y. Liu. 2 females, 1 male, $1 \mathrm{mj}$, locality 1, 6-X-2003; 7 females, 5 males, $2 \mathrm{mj}$, locality 1, 15-I2004; $1 \mathrm{mj}$, locality 3, 27-I-2004; 3 females, 1 males, $1 \mathrm{mj}$, locality 1, 14-IV-2004; 7 females, 9 males, locality 1, 15-VII-2004, coll. Y. Xiong.

Distribution. China (Hainan, Guizhou).

\section{Kenyentulus jinghongensis Yin, 1983}

Material examined. 3 females, locality 1, 25-XI-1984, coll. G. T. Jin \& Z. Y. Liu. Distribution. China (Hainan, Yunnan, Guizhou).

\section{Kenyentulus minys Yin, 1983}

Material examined. 2 females, 2 males, locality 1, 19-XI-1984, coll. G. T. Jin \& Z. Y. Liu. Distribution. China (Hainan, Yunnan, Guangxi, Jiangxi) 


\section{Amphientulus sinensis Xiong, Xie \& Yin, 2005}

Material examined. 1 female, locality 1, 17-XII-2002; 1 female, locality 2; 8 females, 4 males, 1 mj, locality 3, 27-I-2004, coll. Y. Xiong.

Distribution. China (Hainan, Guangdong).

\section{Family Sinentomidae Yin, 1965}

Sinentomon erythranum Yin, 1965

Material examined. 1 female, $1 \mathrm{male}, 1 \mathrm{mj}$, locality 5, 26-II-2003; $1 \mathrm{mj}$, locality 1 , 6-X-2003; 7 females, locality 1, 15-VII-2004, coll. Y. Xiong. 1 female, 1 LI, locality 13, 16-X-2017; 1 LI, locality 12, 17-X-2017, coll. Y. Bu.

Distribution. Widely distributed in South China (Hainan, Shanghai, Jiangsu, Zhejiang, Anhui, Fujian, Guangxi, Guangdong, Hunan, Guizhou, Yunnan).

\section{Family Fujientomidae Yin, 1996}

Fujientomon dicestum Yin, 1977

Material examined. 1 female, locality 5, 26-II-2003, coll. Y. Xiong.

Distribution. China (Hainan, Shanghai, Jiangsu, Zhejiang, Anhui, Ningxia).

\section{Family Eosentomidae Berlese, 1909}

\section{Eosentomon actitum Zhang, 1983}

Material examined. 8 females, 6 males, 2 mj, locality 1, XII-1984; 24 females, 24 males, $27 \mathrm{mj}$, locality 3, 23-I-1985, coll. G. T. Jin \& Z. Y. Liu. 2 females, 3 males, locality 8, 22-II-2003, coll. Y. Xiong. 2 females, 3 mj, locality 11, 22-III-2017, coll. Y. Bu.

Distribution. China (Hainan, Guangdong, Sichuan).

\section{Eosentomon hainanense Yin, 1986}

Material examined. 40 females, 33 males, 2 mj, locality 1, 25-XI-1984, coll. G. T. Jin \& Z. Y. Liu. 2 females, VI-1993, locality 1, coll. C. H. Liao. 1 female, 5 males, locality 8, 22-II-2003; 4 females, 1 male, locality 6, 2-III-2003; 1 female, 3 males, $4 \mathrm{mj}$, 
locality 5, 15-VI-2003; 19 female, 15 males, 8 mj, locality 1, 7-X-2003; 33 females, 31 males, $15 \mathrm{mj}$, locality 1, 14-I-2004; 1 male, locality 3, $960 \mathrm{~m}$ elev., 27-I-2004; 8 females, 8 males, $1 \mathrm{mj}$, locality 1, 15-VII-2004, coll. Y. Xiong.

Distribution. China (Hainan, Yunnan).

\section{Eosentomon iban Imadate, 1965}

Material examined. 2 females, 2 males, locality 1, 27-XI-1984, coll. G. T. Jin \& Z. Y. Liu.

Distribution. China (Hainan); Malaysia, Brunei.

\section{Eosentomon jinhongense Yin, 1982}

Material examined. 2 females, 1 male, locality 2, 14-XI-1984, coll. G. T. Jin \& Z. Y. Liu. Distribution. China (Hainan, Yunnan).

\section{Eosentomon margarops Yin \& Zhang, 1982}

Material examined. 1 female, 2 males, 1 mj, locality 5, 26-II-2003; 1 female, 1 male, $2 \mathrm{mj}$, locality 5, 15-VI-2003; 1 female, 2 males, locality 1, 6-X-2003; 6 females, 3 males, $1 \mathrm{mj}$, locality 1, 15-I-2004; 2 females, 1 male, $1 \mathrm{mj}$, locality 2, $820 \mathrm{~m}$ elev., 27-I-2004; 3 females, 2 males, 2 mj, locality 3, 1000 m elev., 27-I-2004; 2 females, 1 male, locality 1, 14-IV-2004; 2 females, locality 1, 15-VII-2004, coll. Y. Xiong. 1 female, locality 10, 23-III-2017, coll. Y. Bu.

Distribution. China (Hainan, Guangdong, Sichuan).

\section{Eosentomon novemchaetum Yin, 1965}

Material examined. 1 female, locality 11, 22-III-2017, coll. Y. Bu.

Distribution. China (Hainan, Shanghai, Jiangsu, Anhui, Jiangxi, Liaoning, Shaanxi)

\section{Eosentomon orientale Yin, 1965}

Material examined. 1 female, locality 1, 25-XI-1984, coll. G. T. Jin \& Z. Y. Liu.

Distribution. Widely distributed in China (Hainan, Shanghai, Jiangsu, Zhejiang, Anhui, Jiangxi, Hubei, Hunan, Guangxi, Guangdong, Sichuan, Chongqing, Guizhou, Ningxia, Shaanxi, Liaoning). 


\section{Eosentomon sakura Imadate \& Yosii, 1959}

Material examined. 5 females, 3 males, locality 1, 25-XI-1984, coll. G. T. Jin \& Z. Y. Liu. 13 females, 7 males, $9 \mathrm{mj}$, locality 8, 22-II-2003; 1 female, $1 \mathrm{mj}$, locality 5, 20 I-2003; 8 females, 13 males, $7 \mathrm{mj}$, locality 5, 26-II-2003; 1 female, 1 male, locality 6 , 2-III-2003; 2 females, 5 males, $2 \mathrm{mj}$, locality 5, 15-VI-2003; 2 females, $1 \mathrm{mj}$, locality 1, 6-X-2003; 3 females, 1 male, 2 mj, locality 7, 13-VII-2003; 5 females, 2 males, $2 \mathrm{mj}$, locality 1, 15-I-2004; 46 females, 45 males, 29 mj, locality 4, 23-I-2004; 12 females, 20 males, $10 \mathrm{mj}$, locality 2, $820 \mathrm{~m}$ elev., 27-I-2004; 5 females, 5 males, $2 \mathrm{mj}$, locality 1, 500 m elev., 27-I-2004, coll. Y. Xiong. 1 male, locality 2, 20-III-2011, coll. Y. Bu \& C. W. Huang. 8 females, 7 males, 3 mj, locality 11, 22-III-2017, coll. Y. Bu.

Distribution. Widely distributed in China (Hainan, Shanghai, Jiangsu, Zhejiang, Anhui, Jiangxi, Hubei, Hunan, Guangxi, Guangdong, Yunnan, Sichuan, Fujian, Guizhou, Taiwan, Hong Kong, Shaanxi).

\section{Eosentomon shanum (Zhang, 1984)}

Material examined. 2 females, 1 male, locality 4, 23-I-2004; 1 female, 2 males, locality 1, 14-IV-2004; 5 females, $1 \mathrm{mj}$, locality 1, 15-VII-2004, coll. Y. Xiong.

Distribution. China (Hainan, Guangxi, Hunan, Jiangxi).

\section{Eosentomon spanum Yin, 1986}

Material examined. 1 female, 1 male, locality 1, 27-XI-1984, coll. G. T. Jin \& Z. Y. Liu. Distribution. China (Hainan).

\section{Eosentomon tropicum Yin, 1986}

Material examined. 5 females, locality 1, 25-XI-1984, coll. G. T. Jin \& Z. Y. Liu. 1 female, VII-1993, locality 1, coll. C. H. Liao. 1 female, 3 males, 3 mj, locality 8, 22II-2003; 4 females, 3 males, 4 mj, locality 1, 6-X-2003; 2 mj, locality 1, 15-I-2004; 3 females, 1 male, locality 1, 15-VII-2004, coll. Y. Xiong. 2 females, locality 11, 22-III2017, coll. Y. Bu.

Distribution. China (Hainan).

\section{Eosentomon xishaense Yin, 1988}

Material examined. 2 females, locality 1, 27-XI-1984, coll. G. T. Jin \& Z. Y. Liu. 1 mj, VI-1993, locality 1, coll. C. H. Liao. 1 female, locality 5, 20-I-2003; 3 mj, locality 
1, 14-IV-2004; 4 females, 2 males, locality 1, 15-VII-2004, coll. Y. Xiong. 1 female, locality 9, 20-III-2011, coll. Y. Bu \& C. W. Huang. 2 females, 1 male, $1 \mathrm{mj}$, locality 11, 22-III-2017; 1 male, locality 10, 23-III-2017, coll. Y. Bu.

Distribution. China (Hainan, Xisha Islands, Yongxing Island).

\section{Eosentomon yanshanense Yin \& Zhang, 1982}

Material examined. 2 females, locality 1, 25-XI-1984, coll. G. T. Jin \& Z. Y. Liu. 2 females, 4 males, $7 \mathrm{mj}$, locality 6, 2-III-2003; 9 females, 3 males, $10 \mathrm{mj}$, locality 4, 23-I-2004; 2 females, 2 males, locality 1, 14-IV-2004; 2 females, 1 mj, locality 1, 15VII-2004, coll. Y. Xiong.

Distribution. China (Hainan, Guangxi, Guangdong, Fujian, Jiangxi, Hunan, Hubei, Yunnan).

\section{Eosentomon zhanjiangense Zhang, 1983}

Material examined. 2 females, locality 1, 19-I-1985, coll. G. T. Jin \& Z. Y. Liu. 1 females, 2 males, locality 1, 14-IV-2004, coll. Y. Xiong.

Distribution. China (Hainan, Guangdong).

\section{Anisentomon hainanense Xiong, Bu \& Yin, 2008}

Material examined. 1 female, 1 male, locality 1, 6-X-2003, coll. Y. Xiong. Distribution. China (Hainan).

\section{Anisentomon quadrisetum Zhang \& Yin, 1981}

Material examined. 1 female, 1 male, locality 1, 7-X-2003, 14 females; 7 males, locality 1, 14-I-2004, coll. Y. Xiong. 1 male, 1 mj, locality 11, 22-III-2017, coll. Y. Bu.

Distribution. China (Hainan, Guangxi, Guangdong).

\section{Neanisentomon yuenicum Zhang \& Yin, 1984}

Material examined. 1 female, locality 1, 14-IV-2004, coll. Y. Xiong. 1 female, 1 male, locality 10, 23-III-2017, coll. Y. Bu.

Distribution. China (Hainan, Guangdong). 
Paranisentomon tuxeni (Imadate \& Yosii, 1959)

Material examined. 6 females, $1 \mathrm{mj}$, locality 3, $1000 \mathrm{~m}$ elev., 27-I-2004, coll. Y. Xiong. Distribution. China (Hainan, Hubei, Hunan, Jiangxi, Anhui, Guizhou, Shaanxi).

\section{Pseudanisentomon paurophthalmum Zhang \& Yin, 1984}

Material examined. 1 female, $1 \mathrm{mj}$, locality 11, 22-III-2017, coll. Y. Bu.

Distribution. China (Hainan, Guangxi).

\section{Pseudanisentomon molykos Zhang \& Yin, 1984}

Material examined. 5 females, 2 males, $1 \mathrm{mj}$, locality 1, 6-X-2003; 1 female, locality 1, 14-IV-2004, coll. Y. Xiong.

Distribution. China (Hainan, Guangdong, Guangxi, Yunnan).

\section{Pseudanisentomon sininotiale Zhang \& Yin, 1984}

Material examined. 2 females, locality 1, 27-XI-1984, coll. G. T. Jin \& Z. Y. Liu. 2 females, 4 males, locality 4, 23-I-2004; 1 male, locality 3, 600 m elev., 27-I-2004, coll. Y. Xiong.

Distribution. China (Hainan, Guangxi, Hunan).

Discussion. The 34 species of Protura recorded from Hainan Island belong to 11 genera and five families (Protentomidae, Berberentulidae, Sinentomidae, Fujientomidae and Eosentomidae) (Table 5). Most species (91\%) belong to Eosentomidae (21 species) and Berberentulidae (10 species), while the other three families are represented by one species each. Both Berberentulidae and Eosentomidae are widely distributed in China and have high species richness. In contrast, Sinentomidae, Protentomidae, and Fujientomidae each has fewer species occurring in China. Undoubtedly, proturans found in Hainan Island are mainly related to the fauna of Oriental Region and are distinctly different from those from Russian Far East and Siberia (Bu et al. 2014; Shrubovych 2014), which are dominated by the family Acerentomidae.

By comparing the species distribution, we found that the Protura fauna of Hainan Island is closely related to those of neighboring mainland regions (Yin 1999; Szeptycki 2007), and there are 13,10, and 11 species shared with Guangdong, Guangxi, and Yunnan provinces, respectively (Fig. 1; Table 5), which is consistent with the geological history of Hainan Island (Wang 1991; Zhang and Fang 2012). Until the Quaternary period (2.5 million years ago), Hainan Island was still connected with Leizhou Peninsula of Guangdong Province. In the Middle Pleistocene, fault depression led to the separation of Hainan Island from the mainland. With sea level fluctuations, Hainan Island was connected to or separated from the mainland for several times. Since the 
Table 5. The list of proturan species from Hainan Island and their distribution in Hainan Island and three neighboring mainland provinces.

\begin{tabular}{|c|c|c|c|c|c|}
\hline Classification & Species & Hainan & Guangdong & Guangxi & Yunnan \\
\hline \multicolumn{6}{|l|}{ Acerentomata Yin, 1996} \\
\hline \multicolumn{6}{|l|}{ Protentomidae Ewing, 1936} \\
\hline Paracondeellum Yin, Xie \& Zhang, 1994 & P. paradisum sp. n.* & $11^{* *}$ & & & \\
\hline \multicolumn{6}{|l|}{ Berberentulidae Yin, 1983} \\
\hline Baculentulus Tuxen, 1977 & B. tienmushanensis (Yin, 1963) & $2,3,5,11$ & & & + \\
\hline \multirow[t]{8}{*}{ Kenyentulus Tuxen, 1981} & K. ciliciocalyci Yin, 1987 & $1,5,6,9,11,12$ & & & + \\
\hline & K. dolichadeni Yin, 1987 & 2 & & & \\
\hline & K. hainanensis Yin, 1987 & 1,3 & + & & \\
\hline & K. henanensis Yin, 1983 & 2 & & & + \\
\hline & K. japonicus (Imadate, 1961) & 2 & & & + \\
\hline & K. jianfengensis Yin, 1987 & 1,2 & & & \\
\hline & K. jinghongensis Yin, 1983 & 1 & & & + \\
\hline & K. minys Yin, 1983 & 1 & & + & + \\
\hline Amphientulus Tuxen, 1981 & A. sinensis Xiong, Xie \& Yin, 2005 & 1,3 & + & & \\
\hline \multicolumn{6}{|l|}{ Sinentomata Yin, 1996} \\
\hline \multicolumn{6}{|l|}{ Sinentomidae Yin, 1965} \\
\hline Sinentomon Yin, 1965 & S. erythranum Yin, 1965 & $1,5,12,13$ & + & + & + \\
\hline \multicolumn{6}{|l|}{ Fujientomidae Yin, 1996} \\
\hline Fujientomon Yin, 1977 & F. dicestum Yin, 1977 & 5 & & & \\
\hline \multicolumn{6}{|l|}{ Eosentomata Yin, 1996} \\
\hline \multicolumn{6}{|l|}{ Eosentomidae Berlese, 1909} \\
\hline \multirow[t]{14}{*}{ Eosentomon Berlese, 1909} & E. actitum Zhang, 1983 & $1,3,8,11$ & + & & \\
\hline & E. hainanense Yin, 1986* & $1,3,5,6,8$ & + & & + \\
\hline & E. iban Imadate, 1965 & 1 & & & \\
\hline & E. jinhongense Yin, 1982 & 2 & & & + \\
\hline & E. margarops Yin \& Zhang, 1982 & $1,2,3,5,10$ & + & & \\
\hline & E. novemchaetum Yin, 1965 & 11 & & & \\
\hline & E. orientale Yin, 1965 & 1 & + & + & \\
\hline & E. sakura Imadate \& Yosii, 1959 & $1,2,4-8,11$ & + & + & + \\
\hline & E. shanum (Zhang, 1984) & 1,4 & & + & \\
\hline & E. spanum Yin, 1986* & 1 & & & \\
\hline & E. tropicum Yin, $1986^{*}$ & $1,8,11$ & & & \\
\hline & E. xishaense Yin, 1988 & $1,5,9-11$ & & & \\
\hline & E. yanshanense Yin \& Zhang, 1982 & $1,4,6$ & + & + & + \\
\hline & E. zhanjiangense Zhang, 1983 & 1 & + & & \\
\hline \multirow[t]{2}{*}{ Anisentomon Yin, 1977} & A. hainanense Xiong, $\mathrm{Bu} \&$ Yin, 2008* & 1 & & & \\
\hline & A. quadrisetum Zhang \& Yin, 1981 & 1,11 & + & + & \\
\hline Neanisentomon Zhang \& Yin, 1984 & N. yuenicum Zhang \& Yin, 1984 & 1,10 & + & & \\
\hline Paranisentomon Zhang \& Yin, 1984 & P. tuxeni (Imadate \& Yosii, 1959) & 3 & & & \\
\hline \multirow[t]{3}{*}{ Pseudanisentomon Zhang \& Yin, 1984} & P. paurophthalmum Zhang \& Yin, 1984 & 11 & & + & \\
\hline & P. molykos Zhang \& Yin, 1984 & 1 & + & + & \\
\hline & P. sininotiale Zhang \& Yin, 1984 & 1,4 & & + & \\
\hline
\end{tabular}

* Species known only from Hainan Island so far.

** Numbers indicate the localities given in Table 1 .

end of the Quaternary period, due to the drastically rise of sea level, Hainan Island has been separated from the mainland without interruption.

Among the mainland regions neighboring Hainan Island, the Protura fauna of Yunnan Province has been systematically studied (Zhang et al. 1996; Yin et al. 2000), and nearly 80 species were reported from that province, with the Berberentulidae and Ensentomidae having fairly high diversity (Zhang et al. 1996; Yin et al. 2000). In this 
study, we found the diversity of Protura fauna from Hainan Island is very similar to that from Yunnan Province. The only difference is the presence of family Hesperentomidae in Yunnan, which is absent in Hainan Island.

Sampling localities in Hainan Island are still sparse, and additional collection of proturans should be made in the future, so as to reveal the true diversity and provide a better understanding of the biogeography of Protura on the Hainan Island.

\section{Acknowledgements}

We give our cordial gratitude to Mr Gentao Jin, Mr Zuyao Liu, Mr Chonghui Liao, Mr Bowei Tang, and Mr Chengwang Huang for their help in the collection of specimens, to Mr Yiming Yang for his help in preparation of slides, to Mr Rongdong Xie for his identification of part specimens and to Dr Yi Bai for his help in preparation of the map of Hainan Island. We also appreciate Professor José G. Palacios-Vargas for his linguistic corrections to the manuscript as well as his valuable advice. Special thanks are given to Dr Julia Shrubovych and Dr Loris Galli for their valuable comments and review of the manuscript. This research was supported by the National Natural Science Foundation of China (no. 31772509, 31471958 and 31772510), the Natural Science Foundation of Shanghai (no. 17ZR1418700), and the Open Project of Shanghai Key Lab for Urban Ecological Processes and Eco-Restoration (no. SHUES2019A11).

\section{References}

Bu Y, Gao Y, Luan YX, Yin WY (2012) Progress on the systematic study of basal Hexapoda. Chinese Bulletin of Life Sciences 24 (2): 130-138. [in Chinese with English abstract]

Bu Y, Potapov MB, Yin WY (2014) Systematic and biogeographical study of Protura (Hexapoda) in Russian Far East: new data on high endemism of the group. ZooKeys 424: 19-57. https://doi.org/10.3897/zookeys.424.7388

Bu Y, Qian CY, Luan YX (2017) Three newly recorded species of Acerentomata (Hexapoda: Protura) from China, with analysis of DNA barcodes. Entomotaxonomia 39(1): 1-14. https://doi.org/10.11680/entomotax.2017001

Bu Y, Yin WY (2007) Two new species of Hesperentomon Price, 1960 from Qinghai Province, northwestern China (Protura: Hesperentomidae). Acta Zootaxonomica Sinica 32(3): 508-514.

Galli L, Shrubovych J, Bu Y, Zinni M (2018) Genera of the Protura of the world: diagnosis, distribution, and key. ZooKeys 772: 1-45. https://doi.org/10.3897/zookeys.772.24410

Huang FS (2002) Insect Fauna of Hainan Forest. Science Press, Beijing, 1063 pp. [In Chinese] Qian CY, Bu Y, Luan YX (2018) DNA barcoding and an updated key to the genus Hesperentomon (Protura: Acerentomata: Hesperentomidae), with a new species from Northwest China. Zootaxa 4462(4): 523-534. https://doi.org/10.11646/zootaxa.4462.4.5 
Rusek J, Shrubovych J, Szeptycki A (2012) Head porotaxy and chaetotaxy of order Acerentomata (Protura). Zootaxa 3262: 54-61. https://doi.org/10.11646/zootaxa.3262.1.5

Shrubovych J (2014) Identification and character analysis of the Acerentomidae (Protura) of the northeastern Palearctic (Protura: Acerentomidae). Zootaxa 3755(2): 136-164. https:// doi.org/10.11646/zootaxa.3755.2.2

Szeptycki A (2007) Catalogue of the world Protura. Acta Zoologica Cracoviensia 50 (1): 1-210. https://doi.org/10.3409/000000007783995417

Tang B, Yin WY (1988) Three new species of Protura from Sichuan Province. Zoological Research 9(3): 309-315. [in Chinese with English abstract]

Wang XF (1991) Geology of Hainan Island III, Structural Geology. Science Press, Beijing, China, 138 pp. [in Chinese]

Xiong Y (2005) The community diversity of soil animals in the tropical and subtropical forests and the phylogeny of Collembola. PhD Thesis, East China Normal University, Shanghai, 139 pp. [in Chinese with English abstract]

Xiong Y, Bu Y, Yin WY (2008) A new species of Anisentomon from Hainan, Southern China (Protura: Eosentomidae). Zootaxa 1727: 39-43. https://doi.org/10.11646/ zootaxa.1727.1.4

Xiong Y, Xie RD, Yin WY (2005) First record of the genus Amphientulus Tuxen, 1981 (Protura: Acerentomidae) from China, with description of a new species. The Raffles Bulletin of Zoology 53 (1): 1-5.

Yin WY (1986) Three new species and a new record of Eosentomon from Hainan Island, China (Protura: Eosentomidae). Contribution from Shanghai Institute of Entomology 6: 135140. [in Chinese with English abstract]

Yin WY (1987) Four new species of Kenyentulus from hainan Island. Zoological Research 8(2): 149-157. [in Chinese with English abstract]

Yin WY (1999) Fauna Sinica. Arthropoda. Protura. Science Press, Beijing, 510 pp.

Yin WY (2002) Protura. In: Huang FS (Ed.) Insect Fauna of Hainan Forest. Science Press, Beijing, 24-27. [In Chinese]

Yin WY, Xie R, Imadaté G (2000) Protura of Yunnan, Southwest China, with description of four new species (Protura: Eosentomata), In: Aoki J, Yin WY, Imadaté G (Eds) Taxonomical Studies on the Soil Fauna of Yunnan Province in Southwest China, Tokai University Press, Tokyo, 117-131.

Yin WY, Xie R, Zhang J (1994) Phylogeny and biogeography of Condeellum group. (Protura: Protentomidae). Entomologia Sinica 1(3): 195-240. https://doi. org/10.1111/j.1744-7917.1994.tb00245.x

Yin ZW, Li LZ, Wu C (2015) New and little known species of Zorotypus Silvestri (Zoraptera: Zorotypidae) from China. Zootaxa 4007(4): 557-566. https://doi.org/10.11646/ zootaxa.4007.4.6

Zhang J, Xie R, Yin WY (1996) Study on diversity of Protura from Yunnan province. Zoological Research 17(2): 139-146. [in Chinese with English abstract]

Zhang LS, Fang XQ (2012) Paleogeography of China, the Formation of Natural Environment in China. Science Press, Beijing, 425 pp. [In Chinese] 